\title{
Adsorption of Triton $X-100$ and cetyltrimethylammonium bromide mixture with ethanol at nylon-6-solution interface with regard to nylon-6 wettability: II. Work of adhesion and activity of surfactants at interfaces
}

\author{
Magdalena Bielawska $\cdot$ Bronisław Jańczuk • \\ Anna Zdziennicka
}

Received: 17 October 2012/Accepted: 24 December 2012/Published online: 12 January 2013

(C) The Author(s) 2013. This article is published with open access at Springerlink.com

\begin{abstract}
On the basis of the values of the surface tension of the aqueous solutions of the Triton X-100 and CTAB mixture with ethanol, the surface tension of nylon- 6 and the nylon-6-solution interfacial tension, the activity of the surfactant mixture and ethanol at the nylon-6-solution interface was calculated and compared to that at the solution-air one. For these calculations, the Sprow and Prausnitz equation was applied. The obtained values of the activity were used for the calculations of the work of adhesion of the solution to the polymer surface. The values of the work of adhesion obtained in this way were compared to those determined from the Young-Dupre equation by using the contact angle values of the aqueous solutions of the TX-100 and CTAB mixture with ethanol measured on the nylon- 6 surface. The changes of the work of adhesion determined from the Young-Dupre equation were also considered as a function of the surface tension of the solution, its polar component and the interfacial interaction parameter.
\end{abstract}

Keywords Contact angle - Wettability - Critical surface tension of solid wetting - Surface tension - Surfactants . Adhesion work · Nylon-6

Electronic supplementary material The online version of this article (doi:10.1007/s10450-012-9466-3) contains supplementary material, which is available to authorized users.

M. Bielawska · B. Jańczuk · A. Zdziennicka $(\square)$

Department of Interfacial Phenomena, Faculty of Chemistry,

Maria Curie-Skłodowska University, Maria Curie-Skłodowska

Sq. 3, 20-031 Lublin, Poland

e-mail: aniaz@hektor.umcs.lublin.pl

\section{Introduction}

The wettability of solids by the aqueous solutions of the mixtures of surface active agents, which plays a highly important role in many technological processes and everyday life, depends on the activity of the adsorbed surface active agents at different interfaces (Rosen 2004; Tadros 1994). The amount of the adsorbed surface active agents and their activity at the interface depends on the components and parameters of the surface tension of both a given solid and surface active agents.

In the previous paper (Bielawska et al. 2013), the wettability of nylon- 6 by the aqueous solutions of TX-100 and $\mathrm{CTAB}$ mixture with ethanol was considered. It was deduced that the critical surface tension of nylon- 6 wetting $\left(\gamma_{C}\right)$ (Bielawska et al. 2013) is considerably lower than its surface tension and even the Lifshitz-van der Waals component of this tension (Szymczyk et al. 2012) and depends on the way of its evaluation. If $\gamma_{C}$ is determined at the constant concentration of the TX-100 and CTAB mixture, it practically has a constant value in the whole range of the concentration and composition of the surfactant mixture. In the case of the constant ethanol concentration, it appeared that $\gamma_{C}$ changes as a function of its concentration and depends also on the composition of the TX-100 and CTAB mixture.

However, the value of the critical surface tension of nylon-6 wetting obtained at the constant ethanol concentration close to that when complete spreading of the solution over the nylon- 6 surface was observed is nearly the same as that determined at the constant concentration of the surfactant mixture.

It was suggested that the considerable difference between the critical surface tension of nylon- 6 wetting and its surface tension results from the presence of ethanol film 
formed around the settled drop of solution whose pressure was proportional to that at the solution-air interface. This suggestion was confirmed by the calculations of the Gibbs surface excess concentration of ethanol at the nylon6-solution and nylon-6-air interfaces as well as the comparison of the ratio between the difference of the surface excess concentration of ethanol at the nylon-6-air and nylon-6-solution interfaces to that at the solution-air one to the slope of the linear dependence between the adhesion tension and the surface tension of the aqueous solutions of the TX-100 and CTAB mixture with ethanol (Bielawska et al. 2013).

The slope of the $\gamma_{L V} \cos \theta-\gamma_{L V}$ plot for nylon-6 depends on the composition and concentration of the TX-100 and CTAB mixture (Bielawska et al. 2013). This indicates that there should be a direct correlation between the activity of surfactants and ethanol adsorbed at the solution-air and nylon-6-solution interfaces, the interaction parameter across the nylon-6-solution interface, the critical surface tension of nylon- 6 wetting and the work of adhesion of the aqueous solution of the surfactant mixture with ethanol to the nylon-6 surface. Such kind of analysis of the properties of the nylon-6-the solution of the surfactant mixture with ethanol-air systems is difficult to find in the literature but it is highly important from the theoretical and practical points of view.

Thus, the purpose of our studies was to find the correlation between the work of adhesion of the aqueous solutions of the CTAB and TX-100 mixture with ethanol to the nylon-6 surface, the critical surface tension of nylon- 6 wetting, the surface tension of the solution, the solidsolution interfacial tension, the interfacial interaction parameter and the activity of all constituents of the solutions at the solution-air and nylon-6-solution interfaces. For this study, the data from the measurements of the contact angle and the surface tension of the aqueous solutions of the TX-100 and CTAB mixture with ethanol were applied (Bielawska et al. 2012, 2013).

\section{Discussion}

2.1 Activity of water, ethanol and mixture of surfactants at the solution-air and nylon-6-solution interfaces

The surface tension of the aqueous solutions of TX-100 and $\mathrm{CTAB}$ mixture with ethanol and the nylon-6-solution interfacial tension can be predicted on the basis of the activity of the solution components in the bulk phase and in the surface region, as well as their molar surface area (Butler 1932). The relationships between the surface tension of the solution or the solid-solution interfacial tension and the activity of the solution components can be obtained by taking the definition of the chemical potentials of each component in the bulk and surface phases into account.

However, it should be noted that the activity of a given component of the solution can be defined in two different ways: symmetrical $(a)$ and asymmetrical $\left(a^{*}\right)$. Thus, $a$ for a solvent and a solute approaches unity if $X_{i} \rightarrow 1$ ( $X_{i}$ is the mole fraction of the solvent or the solute), and $a^{*}=X_{i}$ for the solvent when $X_{i} \rightarrow 1$ but for the solute when $X_{i} \rightarrow 0$. In the case of the aqueous solutions of surfactant mixture with ethanol, the dependence between their surface tension and the activity of water, surfactant mixture and ethanol in the surface layer and in the bulk phase can be expressed, among others, by the Sprow and Prausnitz equation (Sprow and Prausnitz 1967) which for the solution-air interface $(L A)$ has the following forms:

$$
\begin{aligned}
& \gamma_{L V}=\gamma_{W}+\frac{R T}{\omega_{W}} \ln \frac{a_{W}^{S(L A)}}{a_{W}^{B}} \\
& \gamma_{L V}=\gamma_{A}+\frac{R T}{\omega_{A}} \ln \frac{a_{A}^{S(L A)}}{a_{A}^{B}} \\
& \gamma_{L V}=\gamma_{S}+\frac{R T}{\omega_{S}} \ln \frac{a_{S}^{S(L A)}}{a_{S}^{B}}
\end{aligned}
$$

In the case of the nylon-6-solution interface $(S L)$ we can write:

$$
\begin{aligned}
& \gamma_{S L}=\gamma_{S W}+\frac{R T}{\omega_{W}} \ln \frac{a_{W}^{S(S L)}}{a_{W}^{B}} \\
& \gamma_{S L}=\gamma_{S A}+\frac{R T}{\omega_{A}} \ln \frac{a_{A}^{S(S L)}}{a_{A}^{B}} \\
& \gamma_{S L}=\gamma_{S S}+\frac{R T}{\omega_{S}} \ln \frac{a_{S}^{S(S L)}}{a_{S}^{B}}
\end{aligned}
$$

where $\gamma_{L V}, \gamma_{W}, \gamma_{A}, \gamma_{S}$ is the surface tension of the solution (aqueous solution of the CTAB and TX-100 mixture with ethanol), water, ethanol and surfactant mixture, respectively; $R$ is the gas constant; $T$ is the temperature; $\gamma_{S L}, \gamma_{S W}$, $\gamma_{S A}, \gamma_{S S}$, is the interfacial tension at the nylon-6-solution, nylon-6-water, nylon-6-ethanol and nylon-6-surfactant mixture interface, respectively; $\omega_{W}, \omega_{A}$ and $\omega_{S}$ is the molar area per one square meter of water, ethanol and surfactant mixture at the solution-air and nylon-6-solution interfaces; $a_{W}^{S}, a_{A}^{S}$ and $a_{S}^{S}$ is the activity of water, ethanol and surfactant mixture in the surface layer; $a_{W}^{B}, a_{A}^{B}$ and $a_{S}^{B}$ is the activity of water, ethanol and surfactant mixture in the bulk phase, respectively.

For the surfactant mixture it is difficult to apply (3) or (6) for the calculations of its activity because of its very 
low concentration in the bulk phase as well as the difficulties to establish the molar area of surfactants at the interfaces resulting from the changes of their orientation at a given interface as a function of its concentration in the bulk phase. It is known that $\sum_{i=1}^{\alpha} a_{i}=1$ if the symmetrical definition of the activity is considered and in this case the activity of the surfactant mixture can be determined on the basis of (1) and (2) or (4) and (5), respectively. If the adsorption process of the surfactant mixture at the solution-air and nylon-6-solution interfaces is considered for a dilute solution, it can be assumed, at first approximation, that the activity of surfactants in the bulk phase is very small and that of water is close to the difference between unity and the activity of ethanol.

For the calculation of the activity of water and ethanol from (1) and (2) or (4) and (5), the molar area of water was assumed to be equal to $0.6023 \times 10^{5} \mathrm{~m}^{2} / \mathrm{mol}$ and ethanol $1.26 \times 10^{5} \mathrm{~m}^{2} / \mathrm{mol}$ (Zdziennicka 2010).

The activity of the surfactant mixture was calculated from the expressions

$a_{S}^{S(L A)}=1-a_{A}^{S(L A)}-a_{W}^{S(L A)}$ or $a_{S}^{S(S L)}=1-a_{A}^{S(S L)}-a_{W}^{S(S L)}$

The activity of ethanol and the mixture of TX-100 and CTAB is quite different at the solution-air and nylon-6solution interfaces for the same systems (Figs. 1, 2, S1-S6). The activity of ethanol in the range of its concentration in the bulk phase in which the Young's equation is fulfilled is

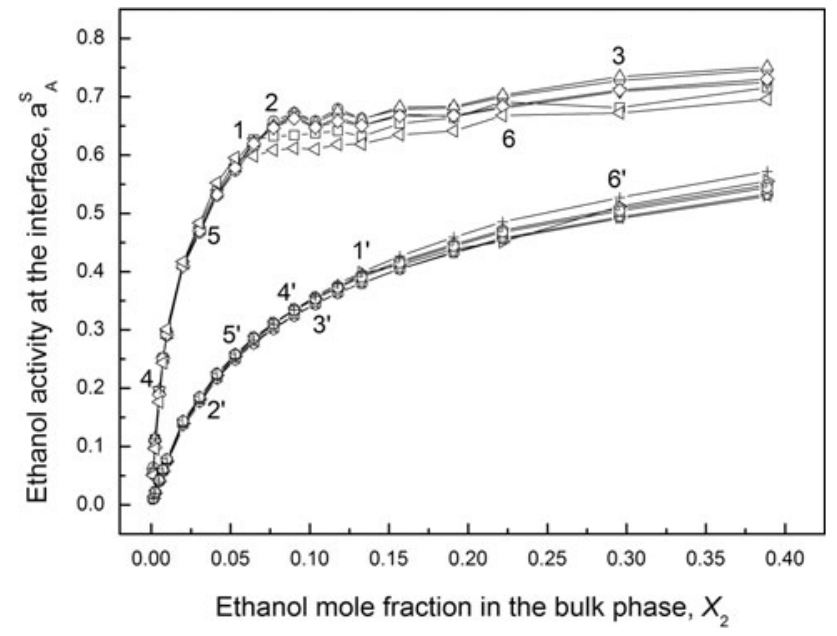

Fig. 1 The dependence between the activity of ethanol at the solution-air (curves 1-6, calculated from (2)) and nylon-6-solution (curves $1^{\prime}-6^{\prime}$, calculated from (5)) interfaces $\left(a_{A}^{S}\right)$ and ethanol mole fraction in the bulk phase $\left(X_{2}\right)$ at the constant total concentration of the TX-100 and CTAB mixture equal to $1 \times 10^{-6} \mathrm{M}$. Curves 1 and $1^{\prime}, 2$ and $2^{\prime}, 3$ and $3^{\prime}, 4$ and $4^{\prime}, 5$ and $5^{\prime}, 6$ and $6^{\prime}$ correspond to the TX-100 mole fraction in the mixture with CTAB equal to $0,0.2,0.4$, $0.6,0.8$ and 1 , respectively lower at the nylon-6-solution interface than at the solutionair one (Figs. 1, S1-S3). The water molecules interact considerably stronger with the nylon- 6 surface than the ethanol ones. The work of adhesion of water to the nylon- 6 surface is equal to $97.6 \mathrm{~mJ} / \mathrm{m}^{2}$ and ethanol to $61 \mathrm{~mJ} / \mathrm{m}^{2}$ (Bielawska et al. 2013). It means that ethanol molecules cannot remove those of water from the nylon-6 surface. In the case of the CTAB and TX-100 mixture at its low concentration, the average activity of that surfactant mixture in the layer at the nylon-6-solution interface is somewhat higher than at the solution-air one (Fig. 2). If the concentration of the TX-100 and CTAB mixture corresponds to its saturated monolayer at both interfaces (Figs. S4-S6) (Bielawska et al. 2013; Szymczyk and Jańczuk 2007), the activity of this mixture at the nylon6-solution interface is lower than that at the solution-air one. It is probably caused by different orientation of the surfactant molecules at the solution-air and nylon6-solution interfaces. At the nylon-6-solution interface, the surfactant molecules take probably the parallel orientation but at the solution-air one they are oriented perpendicularly or at a certain angle to the surface. The higher activity of the surfactant mixture at the nylon-6-solution interface at its low concentration in the bulk phase results probably from very strong interactions of CTAB with the nylon-6 surface. The work of CTAB adhesion to the nylon- 6 surface is equal to $132.4 \mathrm{~mJ} / \mathrm{m}^{2}$ and it is higher than that of water $\left(97.6 \mathrm{~mJ} / \mathrm{m}^{2}\right)$ (Bielawska et al. 2013), therefore the adsorption of CTAB is higher at the nylon-6-solution interface than at the

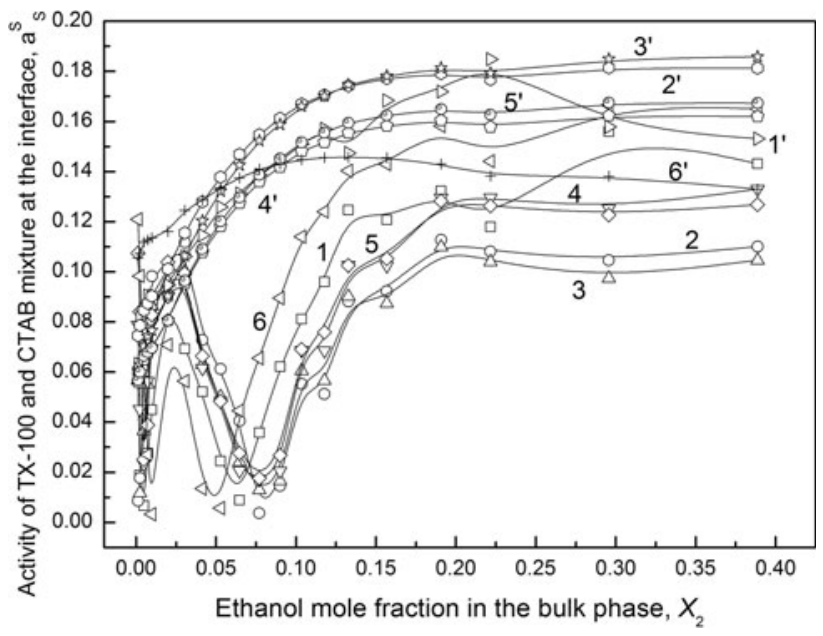

Fig. 2 The dependence between the activity of the TX-100 and CTAB mixture at the solution-air (curves 1-6) and nylon-6-solution (curves $1^{\prime}-6^{\prime}$ ) interfaces calculated from (7) $\left(a_{S}^{S}\right)$ and ethanol mole fraction in the bulk phase $\left(X_{2}\right)$ at the constant total concentration of the TX-100 and CTAB mixture equal to $1 \times 10^{-6} \mathrm{M}$. Curves 1 and $1^{\prime}, 2$ and $2^{\prime}, 3$ and $3^{\prime}, 4$ and $4^{\prime}, 5$ and $5^{\prime}, 6$ and $6^{\prime}$ correspond to the TX-100 mole fraction in the mixture with $\mathrm{CTAB}$ equal to $0,0.2,0.4$, $0.6,0.8$ and 1 , respectively 
solution-air one at low concentration of the surfactant mixture. The work of TX-100 adhesion to the nylon- 6 surface is lower $\left(71.3 \mathrm{~mJ} / \mathrm{m}^{2}\right)$ than that of water and its molecules are probably adsorbed together with the CTAB ones (Bielawska et al. 2013). The composition of the surfactant mixture affects the activity of ethanol only to a low degree. The TX-100 and CTAB mixture at its concentration equal to $1 \times 10^{-4} \mathrm{M}$ behaves somewhat in a different way (Fig. S2). At this concentration, the mixed micelles of surfactants are probably formed at some compositions of the surfactant mixture (Szymczyk and Jańczuk 2007) and therefore the bigger difference between the activity of ethanol at different compositions of the surfactant mixture is observed. The influence of ethanol on the activity of the TX-100 and CTAB mixture is more evident than that of the surfactant mixture on the activity of alcohol. It is interesting that at the ethanol concentration corresponding to the maximum of its surface excess concentration at the solution-air interface, the minimum on the isotherm of the activity of surfactant mixture is observed. The depth of the minimum lowers with the increase of the concentration of the surfactant mixture and at high concentration of the surfactant mixture the minimum of activity does not occur. It is interesting that the changes of the difference between the activity of ethanol in the surface layer and in the bulk phase at the solution-air and nylon-6-solution interfaces as a function of ethanol mole fraction (Figs. 3, S7-S9) are somewhat similar to those of the surface excess concentration (Bielawska et al. 2013) of ethanol at these interfaces.

\subsection{Work of adhesion of the aqueous solutions}

of the TX-100 and CTAB mixture with ethanol to the nylon-6 surface

The adsorption of surfactants at the solid-solution and solution-air interfaces affects strongly the work of adhesion of the solution to the solid surface $\left(W_{a}\right)$. The relationship between the work of adhesion and the surface tension of the phases being in contact has the following form (Adamson and Gast 1997):

$W_{a}=\gamma_{L V}+\gamma_{S V}-\gamma_{S L}$

On the other hand, in the case of the solid-solution drop-air systems, the work of adhesion fulfills the YoungDupre equation (Rosen 2004; Adamson and Gast 1997) which has the following form:

$W_{a}=\gamma_{L V}(\cos \theta+1)$

If we take the presence of the liquid film on the solid surface into account, then (9) has the following form (Adamson and Gast 1997):

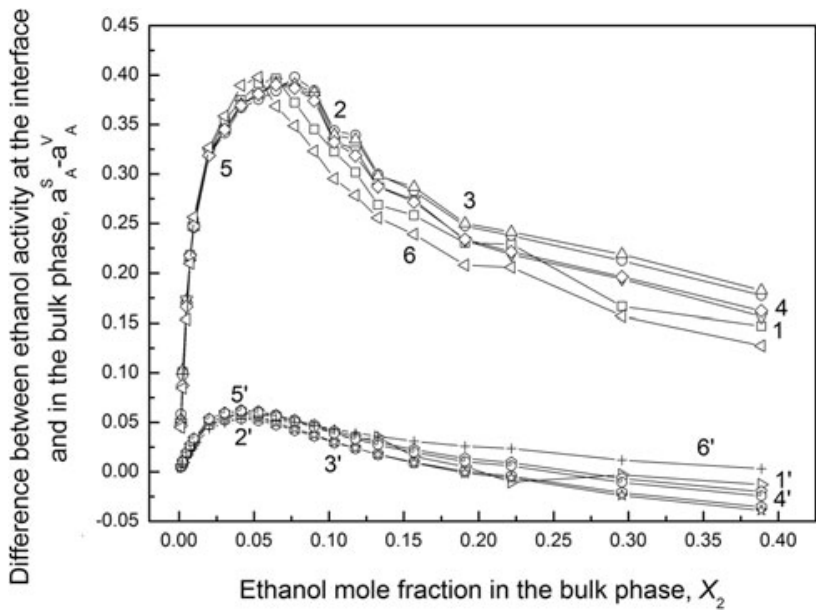

Fig. 3 A plot of the difference between the activity of ethanol at the solution-air interface and in the bulk phase (curves 1-6) as well as between that of ethanol at the nylon-6-solution interface and in the bulk phase (curves $\left.1^{\prime}-6^{\prime}\right)\left(a_{A}^{S}-a_{A}^{V}\right)$ versus ethanol mole fraction in the bulk phase $\left(X_{2}\right)$ at the constant total concentration of the TX-100 and CTAB mixture equal to $1 \times 10^{-6} \mathrm{M}$. Curves 1 and $1^{\prime}, 2$ and $2^{\prime}, 3$ and $3^{\prime}, 4$ and $4^{\prime}, 5$ and $5^{\prime}, 6$ and $6^{\prime}$ correspond to the TX-100 mole fraction in the mixture with $\mathrm{CTAB}$ equal to $0,0.2,0.4,0.6,0.8$ and 1 , respectively

$W_{a}=\gamma_{L V}(\cos \theta+1)+\Pi_{e}$

where $\Pi_{e}$ is the liquid film pressure on the solid surface.

The work of adhesion of the aqueous solutions of the TX-100 and CTAB mixture with ethanol to the nylon-6 surface calculated from (9) and (9a) decreases considerably as a function of ethanol concentration in the range of alcohol concentration in which it is present in the monomeric form in the bulk phase (Zana 1995; Zdziennicka 2010) and practically does not depend on the composition of the surfactant mixture at its concentration corresponding to its unsaturated monolayer (Bielawska et al. 2013). At the concentration of the TX-100 and $\mathrm{CTAB}$ mixture, at which the formation of their mixed micelles takes place, the influence of the surfactant mixture composition on the work of adhesion of the studied solutions to the nylon-6 surface is evident (Figs. 4, S10-S12).

From (9) it results that (Blake 1984):

$\gamma_{L V} \cos \theta=-\gamma_{L V}+W_{a}$

and that

$\cos \theta=-1+\frac{W_{a}}{\gamma_{L V}}=-1+\frac{2 \gamma_{C}}{\gamma_{L V}}$

or generally:

$\cos \theta=-1+W_{a} \frac{1}{\gamma_{L V}}$ 


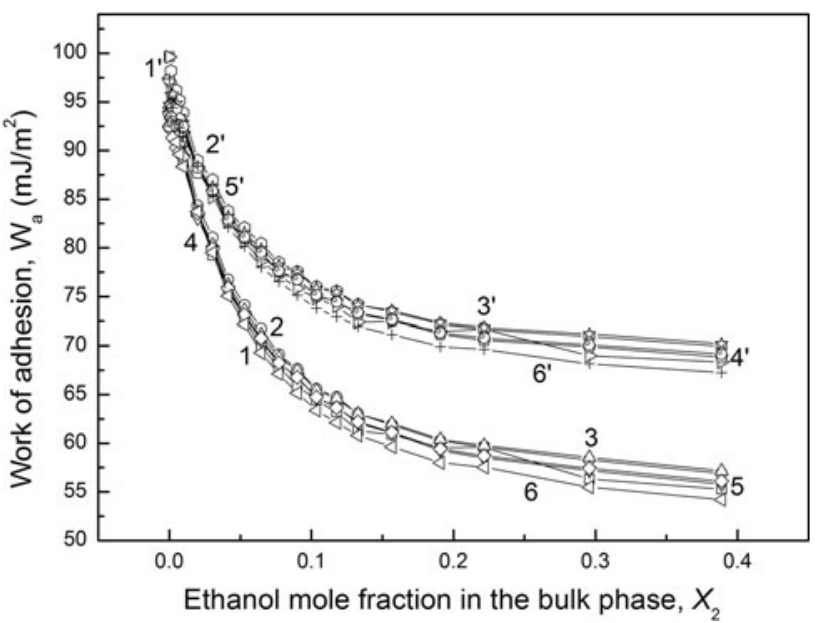

Fig. 4 The dependence between the work of adhesion of the aqueous solutions of the TX-100 and CTAB mixture with ethanol to the nylon6-surface $\left(W_{a}\right)$ and ethanol mole fraction in the bulk phase $\left(X_{2}\right)$ calculated from (9) (curves 1-6) and (9a) (curves $1^{\prime}-6^{\prime}$ ) at the constant total concentration of the TX-100 and CTAB mixture equal to $1 \times 10^{-6} \mathrm{M}$. Curves 1 and $1^{\prime}, 2$ and $2^{\prime}, 3$ and $3^{\prime}, 4$ and $4^{\prime}, 5$ and $5^{\prime}$, 6 and $6^{\prime}$ correspond to the TX-100 mole fraction in the mixture with $\mathrm{CTAB}$ equal to $0,0.2,0.4,0.6,0.8$ and 1 , respectively

Equations (10-12) are fulfilled if the pressure of the liquid film around the drop settled on the solid surface is close to zero.

From (10) it results that if there is a linear dependence between the adhesion tension and the surface tension of the solution whose slope is equal to -1 , then the constant in this dependence is equal to the work of adhesion of the liquid or the solution to the solid surface. In the case when the contact angle of the liquid or the solution on the solid surface is equal strictly to zero, the work of adhesion is equal to the double value of the critical surface tension of solid wetting (Blake 1984). However, from (11) and (12) it results that if the dependence between $\cos \theta$ and the reciprocal of the surface tension is linear and the intercept of the $\cos \theta$ axis is equal to -1 , then the slope of this dependence is equal to the work of adhesion of the liquid or the solution to the solid surface. According to (11), for the liquid or the solution for which the contact angle is equal strictly to zero, the work of adhesion is equal to the work of cohesion $\left(W_{c}\right)$.

Of course, if the conditions mentioned above are fulfilled for the aqueous solutions of surfactants or their mixture then the work of adhesion of the solution to the solid surface is constant and does not depend on the concentration and composition of the surfactant mixture.

For the nylon-6-solution drop-air systems there is a linear dependence between the adhesion tension and the surface tension (Bielawska et al. 2013) as well as between the cosine of the contact angle and the reciprocal of the surface tension of the aqueous solutions of the TX-100 and CTAB mixture with ethanol (Figs. 5, S13-S15) However, the slope of both relationships is larger than -1 and depends on the concentration and composition of the TX-100 and CTAB mixture. For nylon- 6 the relationship between the cosine of contact angle and the reciprocal of the surface tension can be written in the following form:

$\cos \theta=k+l \cdot \frac{1}{\gamma_{L V}}$

where $k$ and $l$ are the constants.

Because the intercept of the cosine axis for nylon- 6 is larger than -1 , therefore, to show the relationship of the slope of the linear dependence between $\cos \theta$ and $\frac{1}{\gamma_{L V}}$ to the work of adhesion, (13) should be presented as (Zdziennicka 2009):

$\cos \theta=-1+\left[(1+k) \cdot \gamma_{L V}+l\right] \cdot \frac{1}{\gamma_{L V}}$

From (14) it results that there is a linear dependence between the work of adhesion of the aqueous solutions of the CTAB and TX-100 mixture with ethanol to the nylon-6 surface and the surface tension of the solution but only in the range of the concentration of the surface active agents in which the Young's equation (Adamson and Gast 1997) is fulfilled. This statement is confirmed in a very wide range of ethanol concentration by the dependence between the work of adhesion of the solution to the nylon-6 surface calculated from (9a) and the surface tension of the solution (Figs. 6, S16-S18) (Bielawska et al. 2012).

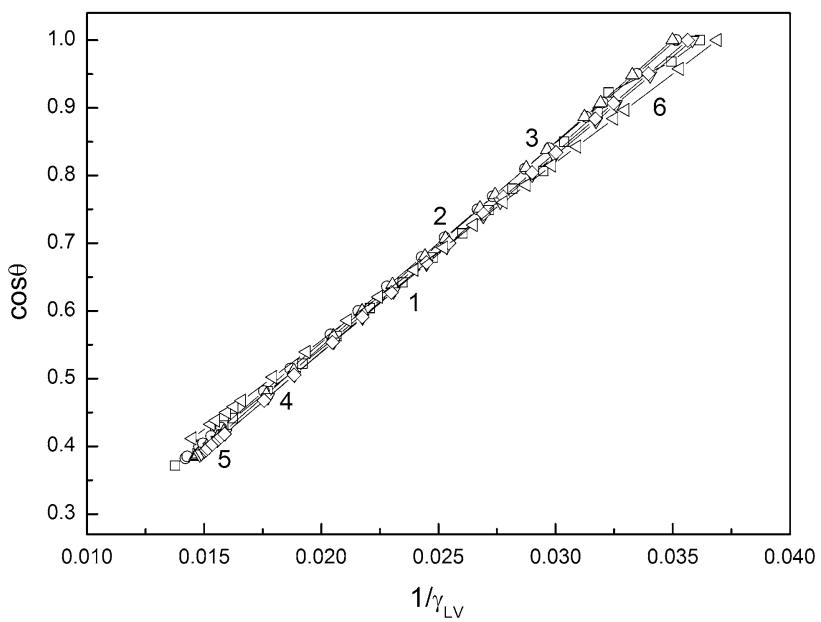

Fig. 5 The dependence between the cosine of the contact angle $(\cos \theta)$ and the reciprocal of the surface tension of the aqueous solution of the TX-100 and CTAB mixture with ethanol $\left(1 / \gamma_{L V}\right)$ at the constant total concentration of the TX-100 and CTAB mixture equal to $1 \times 10^{-6} \mathrm{M}$. Curves $1-6$ correspond to the mole fraction of TX-100 in the mixture with CTAB equal to $0 ; 0.2 ; 0.4 ; 0.6 ; 0.8$ and 1 , respectively 


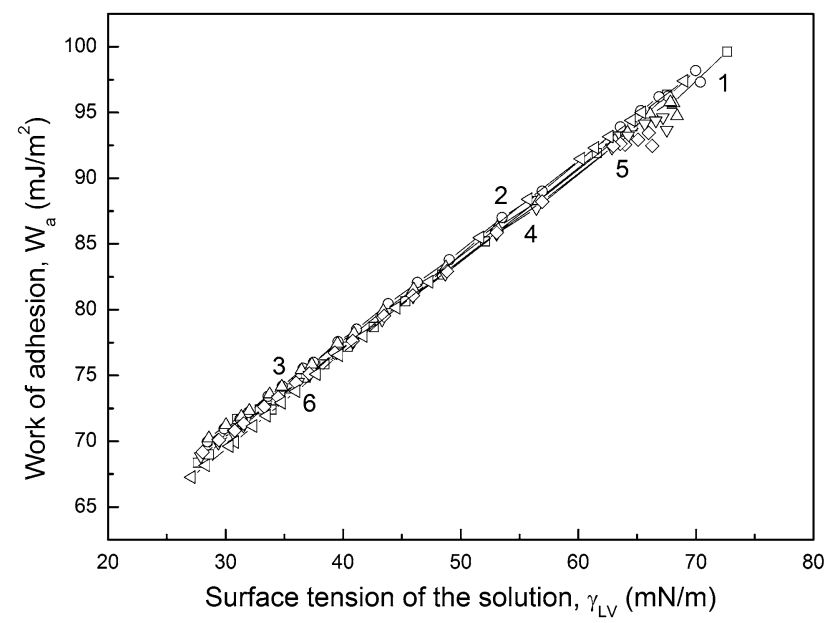

Fig. 6 The dependence between the work of adhesion of the aqueous solutions of the TX-100 and CTAB mixture with ethanol to the nylon6 surface calculated from $(9 a)\left(W_{a}\right)$ and the surface tension of the solution $\left(\gamma_{L V}\right)$ at the constant total concentration of the TX-100 and $\mathrm{CTAB}$ mixture equal to $1 \times 10^{-6} \mathrm{M}$. Curves $1-6$ correspond to the mole fraction of TX-100 in the mixture with $\mathrm{CTAB}$ equal to $0 ; 0.2$; $0.4 ; 0.6 ; 0.8$ and 1 , respectively

If the work of adhesion of the solution to the nylon- 6 surface is a linear function of the solution surface tension, as it results from (8), then the same relationship should occur between the work of adhesion and the surface tension of nylon- 6 covered with the ethanol film as well as the nylon-6-solution interfacial tension. This statement was confirmed by the dependences presented in Figs. 7, 8, S19-S24 also in a wide range of alcohol concentration.

\subsection{Interfacial interaction parameter, activity and work of adhesion}

Taking the Good and Girifalco approach to the interfacial tension into account (Girifalco and Good 1957) in (8) and comparing it to (9), we obtain the expression for the work of adhesion of the liquid to the solid surface in the following form:

$W_{a}=2 \phi \sqrt{\gamma_{S V} \cdot \gamma_{L V}}=\gamma_{L V}(\cos \theta+1)$

where $\phi$ is the interaction parameter across the solid-liquid interface.

For the contact angle equal to zero in (15) we obtain:

$\gamma_{S V}=\frac{\gamma_{C}}{\phi^{2}}$

Taking (16) into account we can state that if the condition for (10) is fulfilled then:

$W_{a}=2 \phi^{2} \gamma_{S V}$

In other cases (17) does not give the reliable values of the work of adhesion. If the surface tension of the solution

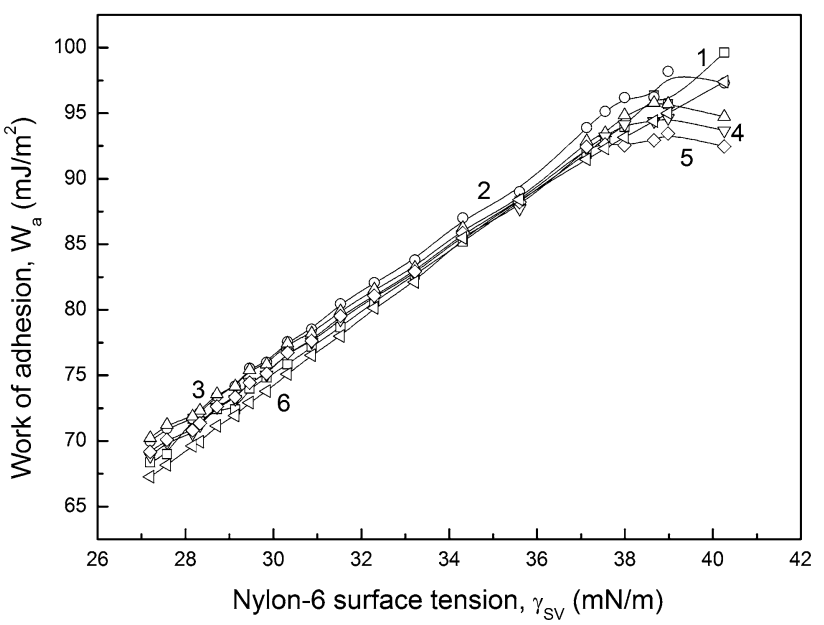

Fig. 7 The dependence between the work of adhesion of the aqueous solutions of the TX-100 and CTAB mixture with ethanol to the nylon6 surface calculated from (9a) $\left(W_{a}\right)$ and the surface tension of nylon-6 covered with the liquid film $\left(\gamma_{S V}\right)$ at the constant total concentration of the TX-100 and CTAB mixture equal to $1 \times 10^{-6} \mathrm{M}$. Curves $1-6$ correspond to the mole fraction of TX-100 in the mixture with CTAB equal to $0 ; 0.2 ; 0.4 ; 0.6 ; 0.8$ and 1 , respectively

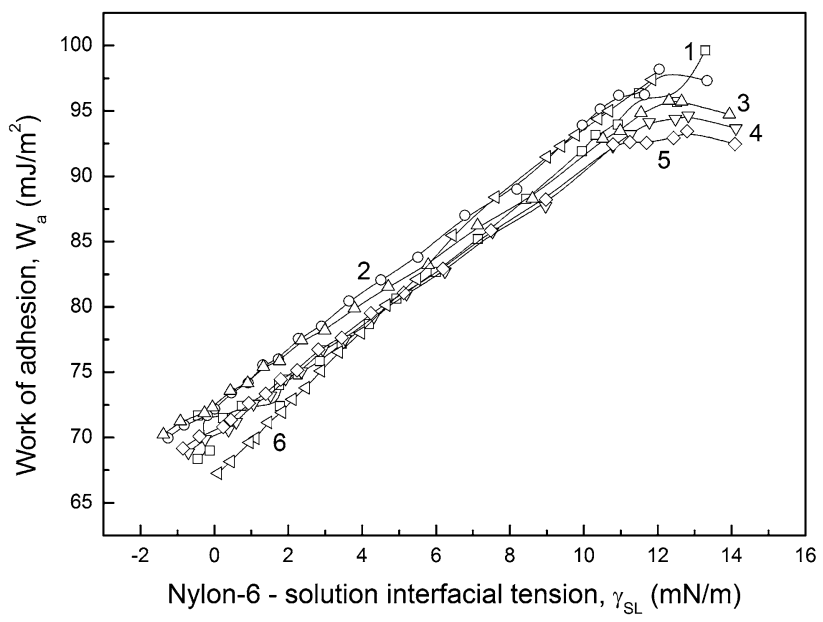

Fig. 8 The dependence between the work of adhesion of the aqueous solutions of the TX-100 and CTAB mixture with ethanol to the nylon6 surface calculated from $(9 a)\left(W_{a}\right)$ and the nylon-6-solution interfacial tension $\left(\gamma_{S L}\right)$ at the constant total concentration of the TX-100 and CTAB mixture equal to $1 \times 10^{-6} \mathrm{M}$. Curves $1-6$ correspond to the mole fraction of TX-100 in the mixture with CTAB equal to $0 ; 0.2 ; 0.4 ; 0.6 ; 0.8$ and 1 , respectively

is equal to the critical surface tension of nylon- 6 wetting by a given series of solutions then combining (13) and (15) we obtain:

$\phi=\sqrt{\frac{l}{\gamma_{S V}(1-k)}}$

The interfacial interaction parameter obtained from (18) depends on the composition of the TX-100 and CTAB mixture and its concentration. Of course, on the assumption 
of the presence or the absence of ethanol film on the nylon6 surface, quite different changes of $\phi$ calculated as a function of ethanol mole fraction are observed (Figs. 9, S25-S27). The values of $\phi$ calculated from (18) have obviously the sense only in the range of ethanol concentration in which the Young's equation is fulfilled. It seems that the values of $\phi$ determined on the assumption that the ethanol film is present on the nylon- 6 surface are more suitable because they approach unity when the surface tension of the aqueous solutions of TX-100 and CTAB mixture with ethanol approaches the critical surface tension of nylon- 6 wetting.

Neumann et al. (1974) showed that for the polymerliquid drop-air systems, the $\phi$ parameter fulfills the following relation:

$\phi=-0.0075 \gamma_{S L}+1$

The values of $\phi$ calculated by combining Eqs. (8) with (15) cannot be described by (19) and the relationship between the parameter $\phi$ and the nylon-6-solution interfacial tension is not linear and depends on the concentration and composition of the surfactant mixture (Figs. 10, S28-S30). According to (15) the $\phi$ parameter is related to the work of adhesion of the solution to the nylon6 surface.

However, there is no linear relationship between $W_{a}$ and the parameter $\phi$ (Figs. 11, S31-S33) in contrast to the linear dependence between the work of adhesion and the surface tension of the solution, the nylon-6-solution interfacial tension or the surface tension of nylon- 6 covered

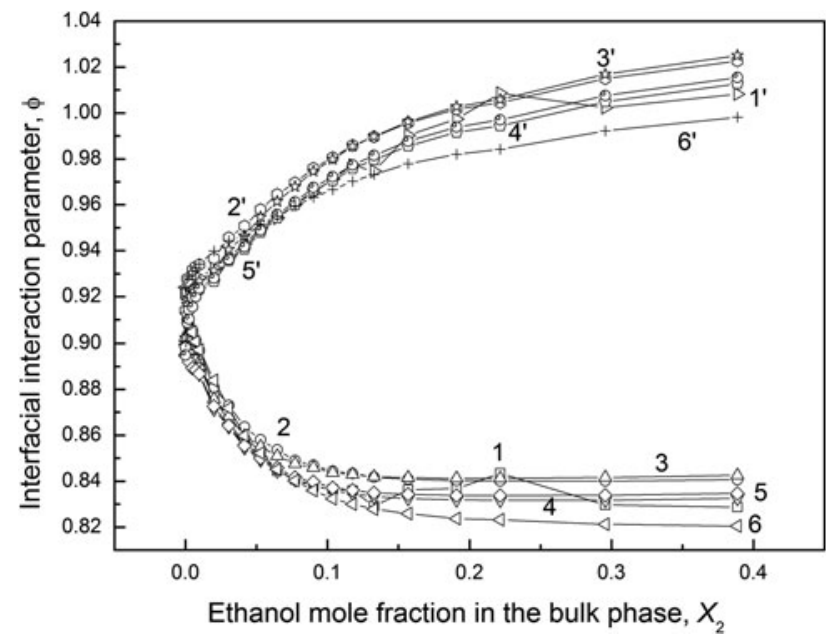

Fig. 9 The dependence between the molecular interaction parameter $(\phi)$ calculated on the assumption of the absence (curves 1-6) and the presence (curves $1^{\prime}-6^{\prime}$ ) of the liquid film on the nylon- 6 surface and ethanol mole fraction in the bulk phase $\left(X_{2}\right)$ at the constant total concentration of the TX-100 and CTAB mixture equal to $1 \times 10^{-6} \mathrm{M}$. Curves 1 and $1^{\prime}, 2$ and $2^{\prime}, 3$ and $3^{\prime}, 4$ and $4^{\prime}, 5$ and $5^{\prime}, 6$ and $6^{\prime}$ correspond to the TX-100 mole fraction in the mixture with $\mathrm{CTAB}$ equal to $0,0.2,0.4,0.6,0.8$ and 1 , respectively

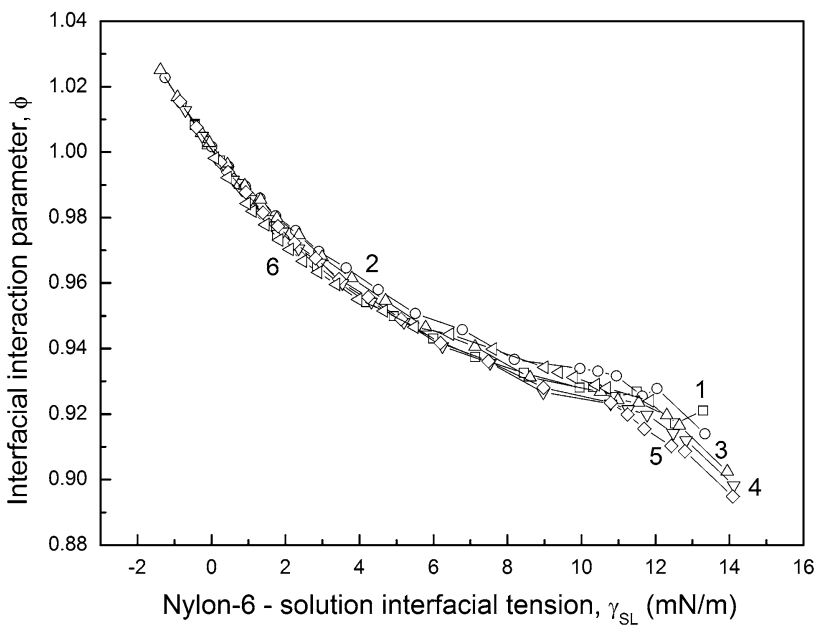

Fig. 10 The dependence between the molecular interaction parameter calculated on the assumption of the presence of the liquid film on the nylon-6 surface $(\phi)$ and the nylon-6-solution interfacial tension $\left(\gamma_{S L}\right)$ at the constant total concentration of the TX-100 and CTAB mixture equal to $1 \times 10^{-6} \mathrm{M}$. Curves $1-6$ correspond to the mole fraction of TX-100 in the mixture with CTAB equal to $0 ; 0.2 ; 0.4 ; 0.6$; 0.8 and 1 , respectively

with the liquid film. The shape of $W_{a}-\phi$ plot depends on the composition of the TX-100 and CTAB mixture and its concentration.

The work of adhesion of the solution should be connected with the work of adhesion of particular constituents of the solution to the nylon- 6 surface if their activity in the surface layer is taken into account. In such a case the work of adhesion of the aqueous solutions of the TX-100 and

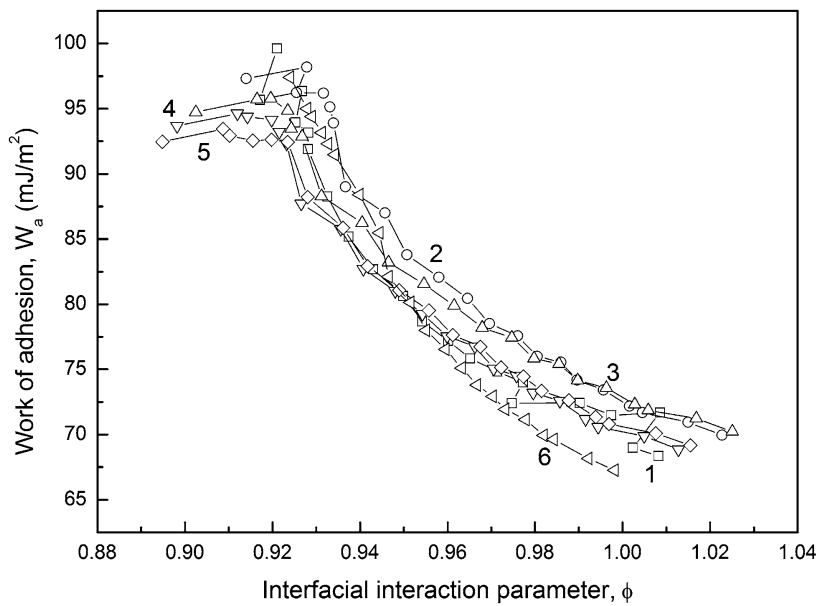

Fig. 11 The dependence between the work of adhesion of the aqueous solutions of the TX-100 and CTAB mixture with ethanol to the nylon- 6 surface calculated from $(9 a)\left(W_{a}\right)$ and the molecular interaction parameter calculated on the assumption of the presence of the liquid film on the nylon-6 surface $(\phi)$ at the constant total concentration of the TX-100 and CTAB mixture equal to $1 \times 10^{-6} \mathrm{M}$. Curves $1-6$ correspond to the mole fraction of TX-100 in the mixture with $\mathrm{CTAB}$ equal to $0 ; 0.2 ; 0.4 ; 0.6 ; 0.8$ and 1 , respectively 
CTAB mixture with ethanol should be described by the following equation:

$W_{a}=a_{W}^{S} \cdot W_{a}^{W}+a_{A}^{S} \cdot W_{a}^{A}+a_{S}^{S} \cdot W_{a}^{S}$

The work of adhesion of the solution to the nylon- 6 surface was calculated from (20) by using the activity of water $\left(a_{W}^{S}\right)$, ethanol $\left(a_{A}^{S}\right)$ and surfactant mixture $\left(a_{S}^{S}\right)$ in the layer at the solution-air and nylon-6-solution interfaces. The work of adhesion of water $\left(W_{a}^{W}\right)$, ethanol $\left(W_{a}^{A}\right)$ and surfactant mixture $\left(W_{a}^{S}\right)$ was calculated from the van Oss et al. (1994) approach to the interfacial tension:

$W_{a}^{i}=2\left(\sqrt{\gamma_{S}^{L W} \cdot \gamma_{L V}^{L W}}+\sqrt{\gamma_{S}^{+} \cdot \gamma_{L V}^{-}}+\sqrt{\gamma_{S}^{-} \cdot \gamma_{L V}^{+}}\right)$

where $\gamma^{L W}$ is the Lifshitz-van der Waals apolar component of the surface tension, $\gamma^{+}$and $\gamma^{-}$are the electron-acceptor and electron-donor parameters of the Lewis acid-base component of the surface tension and the subscripts $S$ and $L V$ refer to the solid and liquid, respectively. For calculations, the values of $\gamma_{S}^{L W}, \gamma_{L V}^{L W}, \gamma_{S}^{+}, \gamma_{L V}^{-}, \gamma_{S}^{-}$and $\gamma_{L V}^{+}$were taken from our previous paper (Bielawska et al. 2013).

The values of $W_{a}$ calculated from (20) are different from those determined from the Young-Dupre equation (Adamson and Gast 1997) independently whether the activity at the solution-air or nylon-6-solution interface was taken for calculations. However, if we assume that ethanol molecules at the nylon-6-air interface are adsorbed on the water film and that only the CTAB molecules can remove the water film from the nylon- 6 surface, then the work of adhesion of the aqueous solutions of the TX-100 and CTAB mixture with ethanol calculated on this basis at low concentration of the surfactant mixture is close to that determined from the Young-Dupre equation (Adamson and Gast 1997) (Fig. S34 as an example). For the calculations, we assumed that the surface tension of nylon- 6 covered with the monolayer of water was equal to $25.6 \mathrm{mN} / \mathrm{m}$ (Zdziennicka and Jańczuk 2010) and that there are only the Lifshitz-van der Waals interactions between such kind of monolayer and ethanol molecules.

It is worth emphasizing that the relationship between the work of adhesion of the solution calculated from the Young-Dupre equation and the ethanol activity at the nylon-6-solution interface for the composition of the surfactant mixture richer in $\mathrm{CTAB}$ can be described by the exponential or linear function.

\section{Conclusions}

On the basis of our considerations dealing with the changes of the work of adhesion of the aqueous solutions of the
TX-100 and CTAB mixture with ethanol to the nylon-6 surface as a function of the surface tension of this solution, the nylon-6 surface tension and the nylon-6-solution interfacial tension, the following conclusions can be drawn:

Although there is a linear dependence between the cosine of the contact angle and the reciprocal of the surface tension of the aqueous solutions of the TX-100 and CTAB mixture with ethanol, its slope is not equal to the work of adhesion of these solutions to the nylon-6 surface.

The work of adhesion of the solution to the nylon- 6 surface calculated from the Young-Dupre equation depends on the concentration and composition of this solution and there is the linear relationship between the work of adhesion and the surface tension of the solution, the nylon-6-solution interfacial tension as well as the surface tension of nylon- 6 covered with the liquid film in the range of ethanol concentration in which the Young's equation is fulfilled.

The work of adhesion of the aqueous solutions of the TX-100 and CTAB mixture to the nylon-6 surface does not depend linearly on the interfacial interaction parameter as it was expected. There is also no linear dependence between this parameter and the nylon-6-solution interfacial tension as it occurs for the "pure" liquids.

However, there is a linear or exponential relationship between the work of adhesion and the activity of ethanol at the nylon-6-solution interface.

Acknowledgments The financial support from the Polish Ministry of Science and Higher Education, Project No. N N204 352040 is gratefully acknowledged.

Open Access This article is distributed under the terms of the Creative Commons Attribution License which permits any use, distribution, and reproduction in any medium, provided the original author(s) and the source are credited.

\section{References}

Adamson, A.W., Gast, A.P.: Physical Chemistry of Surfaces, 6th edn. Wiley, New York (1997)

Butler, J.A.V.: The Thermodynamics of the surfaces of solutions. Proc. R. Soc. A. 138, 348-375 (1932)

Bielawska, M., Jańczuk, B., Zdziennicka, A.: Behaviour of cetyltrimethylammonium bromide, tert-Octylphenol (9.5 EO) ethoxylate and ethanol mixtures at the water-air interface. J. Surfactants Deterg. (2012). doi:10.1007/s11743-012-1379-3

Bielawska, M., Jańczuk, B., Zdziennicka, A.: Adsorption of Triton $\mathrm{X}-100$ and cetyltrimethylammonium bromide mixture with ethanol at nylon-6-solution interface with regard to nylon-6 wettability: I. The effect of adsorption on critical surface tension of nylon-6 wetting. Adsorption (2013). doi:10.1007/s10450012-9465-4

Blake, T.D.: Wetting. In: Tadros, ThF (ed.) Surfactants. Academic Press, London (1984) 
Girifalco, L.A., Good, R.J.: A theory for the estimation of surface and interfacial energies. I. Derivation and application to interfacial tension. J. Phys. Chem. 61, 904-909 (1957)

Neumann, A.W., Good, R.J., Hope, C.J., Sejpal, M.: An equation-ofstate approach to determine surface tensions of low-energy solids from contact angles. J. Colloid Interface Sci. 49, 291-304 (1974)

Rosen, M.J.: Surfactants and Interfacial Phenomena. Wiley Interscience, New York (2004)

Sprow, F.B., Prausnitz, J.M.: Surface thermodynamics of liquid mixtures. Can. J. Chem. Eng. 45, 25-28 (1967)

Szymczyk, K., Zdziennicka, A., Krawczyk, J., Jańczuk, B.: Wettability, adhesion adsorption and interface tension in the polymer/ surfactant aqueous solution system I. Critical surface tension of polymer wetting and its surface tension. Colloids Surf. A: Physicochem Eng Aspects 402, 132-138 (2012)

Szymczyk, K., Jańczuk, B.: The adsorption at solution-air interface and volumetric properties of mixtures of cationic and nonionic surfactants. Colloids Surf. A 293, 39-50 (2007)
Tadros, T.F.: Surfactants in Agrochemicals. Marcel Dekker, New York (1994)

van Oss, C.J.: Interfacial Forces in Aqueous Media. Marcel Dekker, New York (1994)

Zana, R.: Aqueous surfactant-alcohol systems: a review. Adv. Colloid Interface Sci. 57, 1-64 (1995)

Zdziennicka, A.: The wettability of polytetrafluoroethylene and polymethylmethacrylate by aqueous solutions of Triton X-100 and propanol mixtures. Appl. Surface Sci. 255, 7369-7379 (2009)

Zdziennicka, A.: Surface behavior of Triton X-165 and short chain alcohol mixtures. Langmuir 26(2), 1860-1869 (2010)

Zdziennicka, A., Jańczuk, B.: Wettability of quartz by aqueous solution of cationic surfactants and short chain alcohols mixtures. Mat. Chem. Phys. 124, 569-574 (2010) 